\title{
Comparaison de deux méthodes de dosage des acides gras libres totaux du lait de chèvre
}

\author{
G. SELSELET-ATTOU*, Y. CHILLIARD**, \\ P. BAS et P. MORAND-FEHR
}

\section{R és u m é}

L'objet de cette étude est la comparaison de la méthode de Deeth (méthode rapide) et de la méthode de Harper (considérée comme méthode de référence), utilisées pour la détermination des acides gras libres totaux du lait de chèvre, frais ou après $24 \mathrm{~h}$ de stockage au froid. Les résultats obtenus montrent que les pourcentages de récupération des acides gras libres sont relativement plus faibles avec la méthode de Deeth, particulièrement pour les acides gras à courte chaîne. Toutefois, les valeurs absolues d'acidité initiale (lait fraîchement sécrété) enregistrées avec les deux méthodes ne sont pas significativement corrélées, et beaucoup plus élevées avec la méthode de Deeth.

Cependant, les lipolyses (différences entre les teneurs en acides gras libres après $24 \mathrm{~h}$ de stockage à $4^{\circ} \mathrm{C}$, et les teneurs initiales) sont voisines et présentent une corrélation hautement significative. Avec les deux méthodes les coefficients de variation inter-séries sont très élevés pour les faibles lipolyses (inférieures à $200 \mu \mathrm{Eq} / \mathrm{L} / 24 \mathrm{~h}$ ) et varient entre $6 \%$ et $28 \%$ pour des lipolyses plus fortes. Les interférences dues à l'acide lactique et aux phospholipides ont été trouvées faibles ou négligeables dans les laits fraîchement sécrétés et n'expliquent pas entièrement les différences entre les valeurs absolues d'acidité enregistrées par les deux méthodes.

Mots clés : Acides gras libres - Lipolyse - Lait de chèvre - Méthodes de dosage - Acide lactique - Phospholipides.

Laboratoire de Recherche de la Chaire de Zootechnie, Institut National Agronomique - 75005 Paris (France).

* Adresse actuelle : Institut National Agronomique, Département de Technologie des I.A.A., El-Harrach Alger (Algérie).

** Adresse actuelle : Laboratoire de la lactation I.N.R.A.-Theix - 63122 Ceyrat. A qui toute correspondance doit être envoyée. 


\title{
S u m m a r y
}

\author{
COMPARISON OF TWO METHODS FOR MEASURING \\ FREE FATTY ACIDS IN GOAT MILK
}

In this study, Deeth's method (fast method) and Harper's method (considered to be a reference method) were compared for determining total free fatty acids of goat's milk, that was either fresh or kept at $4^{\circ} \mathrm{C}$ for 24 hr.s. Recovery percentages of total free fatty acids (FFA) obtained with Deeth's method were relatively lower than those obtained with Harper's method, especially for short-chain fatty acids (respectively 58 and $92 \%$ for C6). The absolute initial FFA values (freshly-secreted milk) obtained with Deeth's method were on the average more than twice as high as the ones obtained with Harper's. These values were not significatively correlated $(=0,11 ; n=42)$.

However, the calculated lipolysis (estimated from differences between FFA values obtained after 24 hr.s of cold storage and initial FFA values for fresh milk) were similar and highly correlated ( $r=0,99$; $n=42$ ). The inter-series variation coefficients of initial FFA values were relatively high when Harper's method was used with both methods, these inter-series coefficients of variation were found to be very high for the lower lipolysis (lower than or equal to $200 \mu E q / L /$ $24 \mathrm{~h})$. But they varied between 6 and $28 \%$ for higher lipolysis. Interferences due to lactic acid were found to be low or negligible for freshly-secreted milk. Interferences due to phospholipids were lower than $16 \%$ with Deeth's method. Other factors were probably involved in the differences of total acidity obtained with both methods.

Key words: Free fatty acids - Lipolysis - Goat milk - Determination methods - Lactic acid - Phospholipids.

\section{INTRODUCTION}

Les acides gras contenus dans le lait sont principalement sous forme estérifiée dans les triacylglycérols qui constituent $98 \%$ de la matière grasse. Un très faible pourcentage (moins de $1 \%$ ) se trouve sous forme de sel $(\mathrm{RCOONa})$ ou à l'état libre $(\mathrm{RCOOH})$. L'hydrolyse enzymatique des triacylglycérols (lipolyse) conduit à une accumulation dans le lait d'acides gras libres, de mono et diglycérides. Ces composés peuvent être à l'origine d'une altération des qualités organoleptiques du lait et de ses produits dérivés. La détermination de la teneur en acides gras libres est utilisée pour apprécier l'ampleur de la lipolyse dans un lait. Il existe de nombreuses méthodes de dosage qui permettent de mesurer l'évolution de la teneur en acides gras libres totaux, cependant elles ne conduisent pas toutes aux mêmes valeurs (KuzdzalSavoie, 1974 a, b). 
Les méthodes de mesure couramment employées sont basées sur une extraction par solvant des acides gras libres, suivie d'un dosage soit titrimétrique (Frankel et Tarassuk, 1955 ; Harper et coll., 1956 ; Deeth et coll., 1975 ; Salih et coll., 1977) soit colorimétrique (Heeschen et coll., 1975 ; Kuzdzal-Savoie et Trehin, 1975 ; Lindquist et coll., 1975). Plusieurs études comparatives sur les procédés analytiques des acides gras libres totaux du lait ont été effectuées (voir par exemple Stadhouders et coll., 1967 ; Mc Gann et coll., 1975 ; Chilliard et coll., 1983). L'extraction incomplète des acides gras libres, particulièrement des acides gras à courte chaîne, représente la principale difficulté de ces méthodes (Frankel et Tarassuk, 1955 ; Thomas et coll., 1955 ; Kuzdzal-Savoie, 1974 b ; Deeth et coll., 1975 ; Salih et coll., 1977). En effet, en cherchant à améliorer le taux de récupération des acides gras à courte chaîne qui sont hydrophiles, on risque d'augmenter simultanément la récupération des acides organiques tels que l'acide lactique ou l'acide citrique qui peuvent interférer lors du dosage. Par ailleurs, les phospholipides entraînés lors de l'extraction sont susceptibles de causer des interférences avec l'acidité mesurée.

La méthode de Harper et coll. (1956) est généralement considérée comme une méthode de référence. Cependant, cette méthode est relativement longue et délicate à mettre en œuvre et ne peut être utilisée facilement pour un grand nombre d'analyses. Par contre, la méthode de Deeth et coll. (1975) dérivée de la méthode de Dole et Meinertz (1960) présente l'avantage de permettre un travail en série. Par ailleurs, elle est d'une grande simplicité d'exécution et pourrait constituer un moyen de contrôle de la lipolyse au niveau de la ferme et de l'industrie laitière. L'objet du présent travail est de comparer ces deux méthodes et leurs limites d'utilisation pour la détermination des acides gras libres totaux du lait de chèvre.

\section{MATERIEL ET METHODES}

Des chèvres de race Alpine française sont traites manuellement, en évitant le plus possible la contamination microbienne et la formation de mousse. Les mesures d'Acides Gras Libres (AGL) sont réalisées sur des laits fraîchement sécrétés (AGL initiaux) ou lipolysés sous l'action de la lipase naturelle après $24 \mathrm{~h}$ de stockage à $4^{\circ} \mathrm{C}$. Dans certains cas, la lipolyse a été activée par addition de 2 ou $4 \%$ de sérum sanguin de chèvre, dans le but de provoquer une libération importante d'acides gras. Les échantillons de lait frais ou lipolysé sont thermisés à $60^{\circ} \mathrm{C}$ pendant $30 \mathrm{~min}$ dans le but de détruire la lipase naturelle qu'ils renferment, puis conservés à $-50^{\circ} \mathrm{C}$ jusqu'à leur analyse. La lipolyse est égale à la différence entre la teneur finale après $24 \mathrm{~h}$ de stockage à $4^{\circ} \mathrm{C}(\mathrm{AGL} / 24)$ et la teneur initiale $(\mathrm{AGL} / \mathrm{O})$. 


\section{Extraction-titration selon la méthode de Deeth et coll. (1975)}

Les échantillons de lait frais ou lipolysé sont décongelés au bainmarie à $30^{\circ} \mathrm{C}$. Mettre $4 \mathrm{ml}$ de lait dans un tube en verre à col rodé de $35 \mathrm{ml}$ puis ajouter $10 \mathrm{ml}$ de solvant d'extraction à température ambiante. Le solvant d'extraction est le mélange isopropanol : éther de pétrole : acide sulfurique $4 \mathrm{~N}(40: 10: 1)$. Agiter (10 secondes) puis ajouter $6 \mathrm{ml}$ d'éther de pétrole et $4 \mathrm{ml}$ d'eau distillée et agiter de nouveau énergiquement pendant 10 secondes. Laisser décanter $10 \mathrm{~min}$ environ. La titration s'opère à l'aide d'une microburette sur une fraction aliquote $(5 \mathrm{ml})$ du surnageant avec de la potasse méthanolique $0,01 \mathrm{~N}$, sous courant d'azote et en présence d'o-naphtolphtaléine à $1 \%$ ( 2 gouttes) comme indicateur coloré. Un blanc est réalisé par série de vingt échantillons en moyenne, en remplaçant le lait par de l'eau distillée. La lecture du virage de l'indicateur coloré est réalisée à l'œil nu. La teneur en AGL exprimée en $\mu \mathrm{Eq} / 1$ de lait est obtenue d'après la formule suivante :

$$
\begin{aligned}
& \text { AGL en } \mu \mathrm{Eq} / 1=\frac{\mathrm{T} \times \mathrm{N}}{\mathrm{P} \times \mathrm{V}} \times 10^{6} \\
& \mathrm{~T}=\begin{array}{l}
\text { volume de } \mathrm{KOH} \text { versé }(\mathrm{en} \mathrm{ml}) \\
\mathrm{N}=\begin{array}{l}
\text { normalité de la solution de potasse méthanolique (envi- } \\
\text { ron } 0,01 \mathrm{~N}, \text { étalonnée par une solution alcoolique d'acide } \\
\text { palmitique) }
\end{array} \\
\mathrm{P}=\frac{\text { fraction aliquote }(5 \mathrm{ml})}{\text { volume total de surnageant }(9,25 \mathrm{ml} \text { en moyenne) }} \\
\mathrm{V}=\text { volume de lait }(4 \mathrm{ml}) .
\end{array}
\end{aligned}
$$

\section{Extraction-titration selon la méthode de Harper et coll. (1956) modifiée par P. Bas et A. Rouzeau (non publié)}

Les échantillons de lait frais ou lipolysé sont décongelés au bainmarie à $30^{\circ} \mathrm{C}$. L'extraction est réalisée sur $10 \mathrm{ml}$ de lait. Le $\mathrm{pH}$ du lait est ajusté à 1,8-2,0 avec de l'acide sulfurique dans un bécher. Le lait est ensuite mélangé soigneusement à l'aide d'un agitateur de verre avec $17 \mathrm{~g}$ d'acide silicique activé $\left(100^{\circ} \mathrm{C}\right.$ pendant $\left.24 \mathrm{~h}\right)$, de façon à obtenir un mélange homogène sans grumeaux. Puis on ajoute environ 30 à $40 \mathrm{ml}$ du mélange chloroforme-butanol $(95-5, \mathrm{v}-\mathrm{v})$ et l'on homogénise avant de verser et de rincer sur la colonne chromatographique. Celle-ci a un diamètre de $3 \mathrm{~cm}$ et contient, entre 2 morceaux de coton, une suspension chromatographique constituée du mélange chloroforme $(20 \mathrm{ml})$, tampon phosphate $2 \mathrm{M}, \mathrm{pH} 6,5(3 \mathrm{ml})$, acide silicique $(5 \mathrm{~g})$. Les phospholipides et les protéines sont retenus, alors que les acides gras libres et les lipides neutres sont élués par $150 \mathrm{ml} \mathrm{du}$ mélange chloroforme-butanol (qui servent à rincer le bécher). L'éluat est recueilli en fiole sous vide, le vide étant réglé progressivement de 
façon à ce que l'élution soit réalisée en 6 min environ. Après addition de $0,3 \mathrm{ml}$ d'alpha-naphtolphtaléine ( $1 \%$ dans l'éthanol) et de $15 \mathrm{ml}$ d'éthanol distillé à l'éluat, l'acidité totale de celui-ci est titrée par de la potasse éthanolique $0,01 \mathrm{~N}$. Un blanc est réalisé par série de six échantillons, dans lequel le lait est remplacé par de l'eau distillée.

Dans la méthode originale de Harper et coll. (1956) l'indicateur coloré utilisé est du rouge de phénol et le point de virage est déterminé à l'aide d'un colorimètre. Dans le cas de la présente étude la lecture est réalisée à l'œil nu.

\section{RESULTATS ET DISCUSSION}

\section{Récupération des acides gras libres}

Des solutions d'acides gras purs préparées dans les solvants d'extraction employés dans chaque méthode sont incorporées au moment de l'extraction des échantillons de lait. Les taux de récupération sont mesurés pour plusieurs concentrations (200 à $4000 \mu \mathrm{Eq} . / 1$ ) voisines de celles rencontrées dans des laits frais ou lipolysés. Les essais de récupération (tabl. 1) ont été réalisés pour un acide gras à courte chaîne (C6), un acide gras à moyenne chaîne (C13), et un mélange équimolaire de six acides gras (C4, C6, C13, C14, C16, C18 : 1).

Les pourcentages de récupération enregistrés (tabl. 1) avec la méthode de Deeth sont inférieurs à ceux obtenus avec la méthode de Harper, en particulier dans le cas des acides gras à courte chaîne

\section{TABLEAU $1-T A B L E 1$}

Pourcentages de récupération d'acides gras en solution selon la méthode Deeth et la méthode Harper Moyenne (extrêmes)

Recovery percentages of fatty acid solutions with

the Deeth and Harper Méthods Mean

(Lowest and highest values)

\begin{tabular}{c|c|c}
\hline Acides gras & Méthode Deeth & Méthode Harper \\
\cline { 2 - 3 } C6 & $58(56-59)$ & $92(86-96)$ \\
C13 & $85(84-88)$ & $101(97-106)$ \\
$\begin{array}{c}\text { Mélange de six } \\
\text { acides gras: } \\
\text { C4, C13, C14, C16, } \\
\text { C18: 1 (n=6) }\end{array}$ & $81(79-85)$ & $107(101-113)$ \\
\hline
\end{tabular}


(respectivement $58 \%$ et $92 \%$ pour le C6). Cependant les écarts entre les pourcentages de récupération des acides gras à chaîne moyenne (tel que le C13) sont relativement plus faibles (respectivement $85 \%$ et $101 \%)$. Ces résultats sont en accord avec ceux rapportés par différents auteurs (Deeth et coll., 1975 ; Harper et coll., 1956).

Les taux de récupération supérieurs à 100 dans le cas de la méthode de Harper (tabl. 1) pourraient être liés aux erreurs de dosage dues à une plus grande difficulté de lecture causée par le volume important de solvant employé : on utilise en effet quatre fois plus de solvant d'extraction avec la méthode de Harper qu'avec la méthode de Deeth. On peut aussi impliquer des variations éventuelles dues à l'interférence par le $\mathrm{CO}_{2}$ de l'air, bien qu'elles soient normalement prises en compte par les essais à blanc.

\section{Teneurs en acides gras libres initiaux (AGL/O) mesurées selon la méthode de Deeth et la méthode de Harper}

Les valeurs absolues en AGL initiaux (acidité initiale) obtenues selon les deux méthodes ne présentent pas de corrélation significative $(r=0,11$ pour $n=42$ ). Ceci pose un problème d'interprétation de la teneur initiale en AGL. Comme nous le verrons un peu plus loin, lorsque la teneur en AGL est très faible, la reproductibilité des méthodes est très mauvaise.

En outre, les valeurs absolues en acides gras libres initiaux mesurées selon la méthode de Deeth sont en moyenne 2,4 fois plus élevées que celles obtenues avec la méthode de Harper (tabl. 2) ; ce résultat est en accord avec ceux rapportés par Chilliard et coll. (1983) sur lait de vache. La surestimation des AGL initiaux par la méthode de Deeth est sans doute liée à sa technique d'extraction moins spécifique et à l'interférence d'autres acides (voir ci-dessous).

\section{TABLEAU $2-T A B L E 2$}

Teneurs initiales en acides gras libres (AGL/O) mesurées par les deux méthodes (moyenne \pm écart type)

Initial free fatty acid contents $(A G L / O)$ measured with two methods (mean \pm standard deviation)

\begin{tabular}{c|c|c|c}
\hline $\begin{array}{c}\text { Méthode } \\
\text { analytique }\end{array}$ & $\begin{array}{c}\text { Moyennes AGL/O } \\
\text { en } \mu \text { Eq./1 }\end{array}$ & $\begin{array}{c}\text { Corrélation entre } \\
\text { les 2 méthodes }\end{array}$ & $\begin{array}{c}\text { Nombre de } \\
\text { couples }\end{array}$ \\
\hline Deeth & $\begin{array}{c}765( \pm 74) \\
\text { Harper }\end{array}$ & $\mathrm{r}=0,11(\mathrm{NS})$ & 42 laits \\
\hline
\end{tabular}




\section{Relations entre les lipolyses mesurées selon la méthode de Deeth et la méthode de Harper}

Dans cette partie, on ne considère que les évolutions des teneurs en AGL, pendant $24 \mathrm{~h}$ de stockage au froid. Des lipolyses naturelles ou activées par addition de sérum sanguin ont été mesurées par les deux méthodes. L'équation de régression (fig. 1) pour les valeurs de lipolyse (exprimées en $\mu \mathrm{Eq} . / 1 / 24 \mathrm{~h}$ ) est la suivante :

AGL (Deeth) $=0,98$ AGL (Harper) $+51,7$.

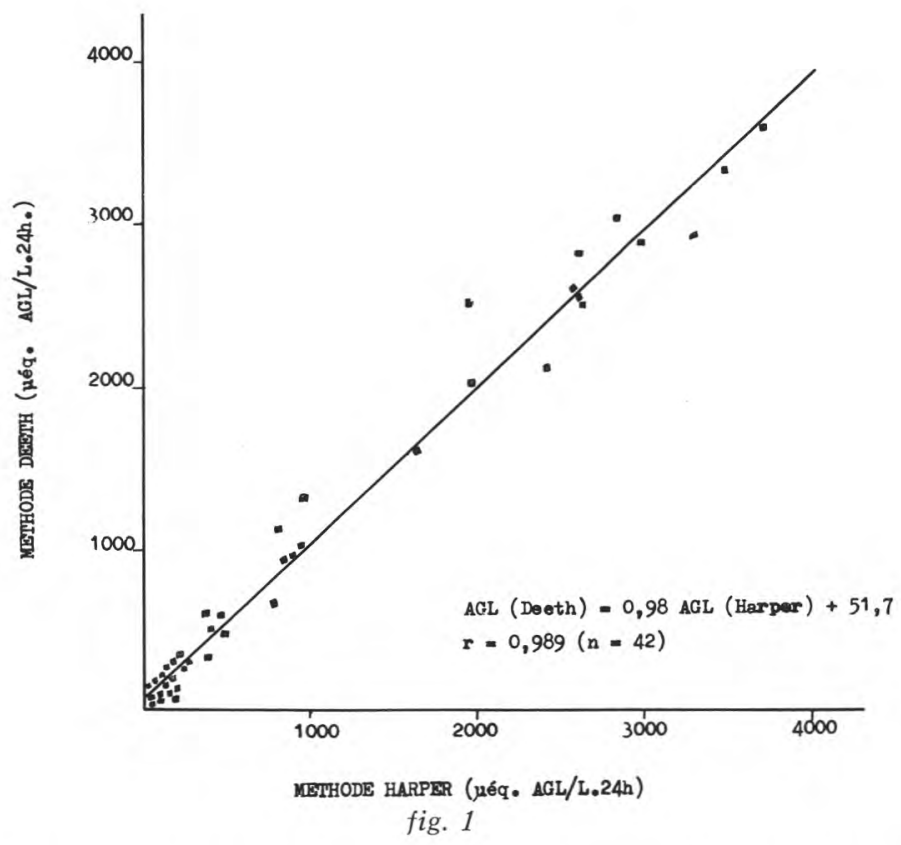

Relations entre les lipolyses mesurées selon la méthode de Deeth et la méthode de Harper

Relationships between lipolysis values obtained with the Deeth and Harper Methods

Le coefficient de corrélation correspondant est élevé et égal à $+0,99$ (pour $n=42$ ). Cette équation est assez stable pour les valeurs de lipolyses inférieures à $1 \mathrm{mEq} . / 1 / 24 \mathrm{~h}(\mathrm{AGL}$ (Deeth) $=1,10 \mathrm{AGL}$ (Harper) +42 , avec $r=0,96$ pour $n=28$ ). Une équation comparable $(\mathrm{AGL}($ Deeth $)=0,84$ AGL (Harper) + 72) mais avec une corrélation plus faible $(r=0,88$ pour $n=29)$ a été observée sur lait de vache par Chilliard et coll. (1983). 


\section{Coefficient de variation inter-séries des résultats de mesure des AGL initiaux (AGL/O) et des lipolyses obtenus selon la méthode de Deeth et la méthode de Harper}

Les coefficients de variation inter-séries des teneurs initiales en AGL enregistrées dans le cas de la méthode Harper sont relativement élevés, comparés à ceux obtenus avec la méthode de Deeth (tabl. 3). Ceci est dû en partie aux valeurs plus élevées observées avec cette dernière méthode (tabl. 2). D'autre part, les coefficients de variation inter-séries pour les faibles lipolyses ( 0 à $500 \mu \mathrm{Eq} . / 1 / 24 \mathrm{~h}$ ) sont plus élevés dans le cas de la méthode de Harper (tabl. 3). Ceci pourrait être en partie dû à l'interférence relative par le $\mathrm{CO}_{2}$ de l'air qui est probablement d'autant plus élevée que la teneur en acides gras libres

\section{TABLEAU $3-T A B L E 3$}

Coefficients de variation inter-séries des résultats de lipolyse et d'AGL initiaux

Inter-series coefficients of variation of lipolysis and initial FFA values

\begin{tabular}{|c|c|c|c|c|c|}
\hline \multirow{2}{*}{\multicolumn{2}{|c|}{$\begin{array}{l}\text { Niveau de lipolyse } \\
\mu \mathrm{Eq} / \mathrm{l} / 24 \mathrm{~h} \\
(\text { ou } \mu \mathrm{Eq} / \mathrm{l})(2)\end{array}$}} & \multicolumn{2}{|c|}{ Méthode de Deeth (1) } & \multicolumn{2}{|c|}{ Méthode de Harper } \\
\hline & & $\begin{array}{l}\text { Coefficient de } \\
\text { variation } \\
\text { inter-séries }\end{array}$ & $\mathrm{n}$ & $\begin{array}{l}\text { Coefficient de } \\
\text { variation } \\
\text { inter-séries }\end{array}$ & $\mathrm{n}$ \\
\hline & $0-200$ & 43,6 & 81 & 68,1 & 8 \\
\hline & $200-500$ & 15,9 & 29 & $\begin{array}{l}28,1 \\
27,4(2)\end{array}$ & $\begin{array}{c}8 \\
16(2)\end{array}$ \\
\hline & $500-1000$ & $\begin{array}{l}11,4 \\
7,6(2)\end{array}$ & $\begin{array}{l}18 \\
80(2)\end{array}$ & - & - \\
\hline & $1000-2000$ & 9,8 & 16 & - & - \\
\hline & $2000-4000$ & 6,4 & 16 & 7,8 & 6 \\
\hline \multicolumn{6}{|c|}{$\begin{aligned} \mathrm{n}= & \text { nombre de laits analysés en double, dans deux séries distinctes de } \\
& \text { mesure. L'écart-type est calculé par la formule S.D. }=\left(\varepsilon \mathrm{di}^{2} / 2 \mathrm{n}\right)^{1} / 2 \text {, où } \\
& \text { di représente la différence entre les doubles. }\end{aligned}$} \\
\hline \multicolumn{6}{|c|}{$\begin{array}{l}\text { (1) Ces résultats ont été confirmés pour les valeurs d'AGL initiaux et de } \\
\text { lipolyse d'un lait, analysé au cours de } 29 \text { séries distinctes d'analyse, } \\
\text { par la méthode de Deeth : } \\
\text { AGL initiaux }=770 \pm 72 \mu \mathrm{Eq} / 1(\mathrm{CV}=9,4 \%) \text {. } \\
\text { Lipolyse }=1871 \pm 194 \mu \mathrm{Eq} / \mathrm{l} / 24 \mathrm{~h}(\mathrm{CV}=10.4 \%) \text {. }\end{array}$} \\
\hline \multicolumn{6}{|c|}{ (2) Valeurs obtenues pour les AGL initiaux. } \\
\hline
\end{tabular}


mesurée est faible. En outre, comme nous l'avons rapporté précédemdent, on rencontre une plus grande difficulté de lecture causée par le volume important de solvant, qui est à l'origine d'une erreur absolue plus importante.

Nous avons par ailleurs cherché à préciser l'importance de l'interférence éventuelle de composés acides contenus dans le lait tels que l'acide lactique et les phospholipides, dans le but d'expliquer la surestimation des AGL initiaux par la méthode de Deeth.

\section{Interférence par l'acide L - lactique}

L'acide L - lactique est extrait selon les méthodes de Deeth et de Harper dans un lait d'acidité connue, auquel on incorpore des concentrations croissantes d'acide $\mathrm{L}$ - lactique voisines de celles rencontrées dans un lait de chèvre frais ou lipolysé $\left(24 \mathrm{~h}\right.$ à $\left.4^{\circ} \mathrm{C}\right)$. La teneur en acide lactique des laits frais de chèvre mesurée selon la méthode enzymatique de Boehringer est en moyenne de $10 \mathrm{mg} / \mathrm{l}$ (Selselet-Attou, 1982).

Les pourcentages d'acide lactique récupéré dans l'extrait en fonction des quantités ajoutées dans le lait (tabl. 4) sont faibles mais relativement plus élevés avec la méthode de Deeth. Les pourcentages d'interférence (augmentation en \% de l'acidité mesurée) pour les additions croissantes d'acide L - lactique sont d'autant plus élevés que la teneur en acides gras libres du lait est faible et que la concentration en acide L - lactique augmente. Enfin, l'interférence due à l'acide lactique ou à d'autres acides organiques peut devenir notable (10 à $30 \%$ des valeurs enregistrées par la méthode de Deeth) pour des teneurs d'environ $300 \mathrm{mg} / 1$ d'acide lactique observées dans des laits conservés durant 36 h à $15^{\circ} \mathrm{C}$ (Selselet-Attou, 1982).

La détermination de l'interférence éventuelle d'autres acides organiques (acides citrique, isocitrique, cétoniques, ...) aurait été utile. Le lait de chèvre renferme en moyenne 0,5 à $2 \mathrm{~g} / 1$ d'acides citrique et isocitrique (Peaker et coll., 1981).

\section{Interférence par les phospholipides avec la méthode de Deeth}

Une estimation de l'interférence par les phospholipides a été réalisée seulement pour la méthode de Deeth, car, pour la méthode de Harper nous avons vérifié par chromatographie sur plaque que la colonne d'acide silicique retient la totalité des phospholipides.

L'estimation de l'interférence par les phospholipides est réalisée à l'aide d'une solution de lécithines de jaune d'œuf préparée dans le solvant d'extraction de la méthode de Deeth, incorporée à concentration croissante dans les échantillons de lait au moment de l'extraction. Les teneurs en acides gras des laits utilisés varient entre 755 et $1293 \mu \mathrm{Eq} . / 1$ (selon la méthode de Deeth). 


\section{TABLEAU $4-T A B L E 4$}

Estimation de l'interférence due à l'acide lactique dans la mesure des AGL du lait par les méthodes de Deeth et de Harper

Evaluation of the interference due to lactic acid during the determination of milk free fatty acids with the Deeth and Harper Methods

\begin{tabular}{|c|c|c|c|c|c|}
\hline \multicolumn{2}{|c|}{$\begin{array}{l}\text { Lactate ajouté } \\
\text { dans le lait }\end{array}$} & \multicolumn{2}{|c|}{$\begin{array}{l}\text { Acidité mesurée } \\
\text { due au lactate ajouté } \\
(\mu \mathrm{Eq} / 1)\end{array}$} & \multicolumn{2}{|c|}{$\begin{array}{l}\text { Interférence (\%) pour } \\
\text { une teneur en AGL de } \\
700 \mu \mathrm{Eq} / \mathrm{l} \text { (1) }\end{array}$} \\
\hline $\mathrm{mM} / 1$ & $\mathrm{mg} / \mathrm{l}$ & $\begin{array}{l}\text { Deeth } \\
(\mathrm{n}=3)\end{array}$ & $\begin{array}{l}\text { Harper } \\
(\mathrm{n}=1)\end{array}$ & Deeth & Harper \\
\hline 0,3 & 27 & 15 & - & 2,1 & - \\
\hline 1,2 & 108 & 4 & 0 & 0,6 & 0 \\
\hline 2,5 & 225 & 7 & - & 1,0 & - \\
\hline 5 & 450 & 87 & - & 12,4 & - \\
\hline 6 & 540 & - & 0 & - & 0 \\
\hline 20 & 1800 & 359 & - & 51,3 & - \\
\hline 30 & 2700 & - & 300 & - & 42,9 \\
\hline 40 & 3600 & 805 & - & 115,0 & - \\
\hline
\end{tabular}

(1) Correspondant à une teneur en AGL initiaux de $300 \mu \mathrm{Eq} / 1$ et à une lipolyse de $400 \mu \mathrm{Eq} / 1 / 24 \mathrm{~h}$ (méthode de Harper).

La teneur moyenne des laits de chèvre en phospholipides est comprise entre 200 et $300 \mathrm{mg} / 1$ selon Jenness (1980). On observe que, même en doublant la teneur en phospholipides par une adjonction de $300 \mathrm{mg} / 1$ de lécithines de jaune d'œuf (tabl. 5), le pourcentage d'interférence (augmentation en \% de l'acidité mesurée) est relativement faible (environ $16 \%$ ) dans un lait frais dont la teneur réelle en acides gras libres serait égale à $300 \mu \mathrm{Eq}$./1. De même que pour l'acide lactique, l'interférence par les phospholipides est d'autant plus élevée que la teneur en acides gras libres du lait est faible et que la concentration en lécithines de jaune d'œuf augmente. Ainsi l'interférence relative des phospholipides dépend de la vraie valeur des AGL à laquelle on la rapporte. Enfin, il aurait été intéressant pour la rigueur du raisonnement d'analyser la teneur en phospholipides des laits expérimentaux au cours de la lactation. En effet, on sait que la teneur en phospholipides peut augmenter de $50 \%$ environ dans le lait de vache et de 


\section{TABLEAU 5 - TABLE 5}

Estimation de l'interférence due aux lécithines dans la mesure des AGL du lait par la méthode de Deeth

Evaluation of interference due to lecithins during the determination of milk free fatty acids with the Deeth Method

\begin{tabular}{|c|c|c|c|}
\hline & $\begin{array}{l}\text { Lécithines } \\
\text { ajoutées au lait } \\
(\mathrm{mg} / \mathrm{l}) \text { (1) }\end{array}$ & $\begin{array}{l}\text { Acidité mesurée } \\
\text { due aux lécithines } \\
\text { ajoutées ( } \mu \mathrm{Eq} / 1)(2)\end{array}$ & $\begin{array}{c}\text { Interférence }(\%) \text { pour } \\
\text { une teneur en AGL } \\
\text { de } 300 \mu \mathrm{Eq} / 1 \text { (3) }\end{array}$ \\
\hline & 100 & 4 & 1 \\
\hline & 200 & 14 & 5 \\
\hline & 300 & 48 & 16 \\
\hline & 400 & 112 & 37 \\
\hline & 500 & 179 & 60 \\
\hline & 600 & 281 & 94 \\
\hline & 700 & 353 & 118 \\
\hline (1) & \multicolumn{3}{|c|}{ Lécithines de jaune d'œuf. } \\
\hline (2) & \multicolumn{3}{|c|}{ Moyenne de 2 déterminations. } \\
\hline (3) & \multicolumn{3}{|c|}{ Valeur moyenne des AGL initiaux estimée par la méthode de Harper. } \\
\hline
\end{tabular}

bufflesse au cours d'une lactation complète (Kuchroo et Narayanan, 1977). L'interférence possible d'autres classes de phospholipides reste en outre à préciser.

Bien que les interférences de l'acide lactique et des lécithines ne soient probablement pas toujours négligeables, elles ne permettent pas d'expliquer totalement la forte surestimation des AGL initiaux systématiquement rencontrée avec la méthode de Deeth. Cette méthode convient donc mieux pour mesurer des évolutions de teneurs en AGL du lait, plutôt que les valeurs absolues de celles-ci.

\section{CONCLUSION}

En conclusion, la méthode de Deeth permet une assez bonne récupération globale des acides gras libres ( $80 \%$ environ). Les pertes enregistrées concernent essentiellement les acides gras à courte chaîne. Contrairement aux acides gras libres initiaux, les lipolyses mesurées selon cette méthode sont voisines et présentent une 
corrélation élevée avec celles obtenues par la méthode de Harper. L'interférence due à l'acide lactique dans un lait de chèvre frais ou conservé 24 h à $4^{\circ} \mathrm{C}$ est faible et celle due aux phospholipides dans le cas de la méthode de Deeth serait inférieure à $15 \%$ de la valeur de référence mesurée par la méthode de Harper. En outre, les erreurs absolues engendrées par ces interférences sont en grande partie supprimées lorsqu'il s'agit de la mesure de la lipolyse, qui constitue une différence entre la teneur finale (après $24 \mathrm{~h}$ de stockage) et la teneur initiale en AGL du lait frais.

Cette étude est essentiellement destinée à fournir des éléments objectifs aux utilisateurs de méthodes de dosage des acides gras libres totaux du lait. La méthode de Deeth semble permettre un contrôle rapide de la lipolyse au niveau de la ferme et de l'industrie laitière mais se prête mal à l'étude des AGL initiaux puisqu'elle les surestime d'environ $140 \%$. Une étude comparative avec d'autres méthodes de référence et d'autres méthodes rapides disponibles reste à conduire sur lait de chèvre.

\section{Bibliographie}

Chilliard (Y.), Bauchart (D.), Cartier (P.) (1983). - Comparaison de 5 méthodes titrimétriques avec une méthode chromatographique de dosage des acides gras libres du lait de vache. ITEB-INRA. Compte rendu d'expérimentation $\mathrm{n}^{\circ} 84031$, p. $2.1-2.15$.

Deeth (H. C.), Fitz-Gerald (C. H.), Wood (A. F.) (1975). - A convenient method for determining the extent of lipolysis in milk. Aust. J. Dairy Technol., $30,109$.

Dole (V.P.), Meinertz (H.) (1960). - Microdetermination of long chain fatty acids in plasma and tissues. J. Biol. Chem., 235, 2595.

Frankel (E. N.), TARASSUK (N. P.) (1955). - An extraction - titration method for the determination of free fatty acids in rancid milk and cream. J. Dairy Sci., 38, 751.

Harper (W. J.), SchWartz (D. P.), El-Hagarawy (I. S.). (1956). - A rapid silicagel method for measuring total free fatty acids in milk. J. Dairy Sci., 39, 46.

Heeschen (W.), Suhren (G.), Tolle (A.) (1975). - Measurement of free fatty acids in milk in the auto analyzer II system. In Proceedings of the lipolysis symposium, Cork. Féd. Int. Laiterie, Doc. 86, 178.

Jenness (R.) (1980). - Composition and characteristics of goat milk. J. Dairy Sci., 63, 1630.

KUCHROO (T. K.), NARAYANAN (K. M.) (1977). - Effect of stage of lactation on distribution of fat globules and phospholipid content of milk. Indian $J$. Dairy Sci., 30, 99-104.

KuzDzal-Savoie (S.) (1974 a). - Assessement of lipolysis in milk. Organoleptic aspects. Féd. Int. Laiterie, Doc. 82, 23.

KuzDZAL-Savoie (S.) (1974 b). - Assessement of lipolysis in milk. Determination of free fatty acids. Féd. Int. Laiterie, Doc. 82, 12.

Kuzdzal-Savore (S.), TREHIN (J.) (1975). - Use of rhodamine for determination of free fatty acids in cow's milk. In Proceedings of the lipolysis symposium, Cork. Féd. Int. Laiterie, Doc. 86, 180. 
Lindouist (B.), Roos (T.), Fujita (H.) (1975). - Autoanalyser determination of free fatty acids in farm milk. Modification of present methods to simplify transportation of the sample. In Proceedings of the lipolysis symposium, Cork. Féd. Int. Laiterie, Doc. 86, 171.

Mc Gann (T. C. A.), Eason (D. C.), Downey (W. K.) (1975). - Comparative evaluation of methods for determining free fatty acids in milk supplies. In Proceedings of the lipolysis symposium, Cork. Féd. Int. Laiterie, Doc. 86, 184.

Peaker (M.), Faulkner (A.), Blatchford (D. R.) (1981). - Changes in milk citrate concentration during lactation in the goat. J. Dairy Res., 48, 357.

Salih (A. M.), Anderson (J.), Tuckley (B.) (1977). - The determination of short and long chain free fatty acids in milk. J. Dairy Res., 44, 601.

Selselet-Attou (G.) (1982). - Caractéristiques du système lipolytique et lipolyse spontanée du lait de chèvre. Thèse Doc. Sci., Université de Paris VI, Paris (France).

Stadhouders (J.), Tuckey (S. L.), RaAdsveld (C. W.) (1967). - Comparison of certain methods for the determination of fat hydrolysis in milk. Neth. Milk Dairy J., 21, 150.

Thomas (E. L.), Nielsen (A. J.), Olson (J. C.) (1955). - Hydrolytic rancidity in milk, a simplified method for estimating the extent of its development. American Milk Review, 17, 50. 